\title{
Synergistic apoptotic effects of apigenin TPGS liposomes and tyroservatide: implications for effective treatment of lung cancer
}

This article was published in the following Dove Press journal:

International Journal of Nanomedicine

17 July 2017

Number of times this article has been viewed

Xin Jin

Qing Yang

Youwen Zhang

Department of Hospital Pharmacy, Suqian Branch Jiangsu Province

Hospital, Suzhilu, Suqian,

People's Republic of China
Correspondence: Xin Jin Department of Hospital Pharmacy, Suqian Branch Jiangsu Province Hospital, I 20 Suzhilu, Suqian 223800, People's Republic of China Email jinxin87।21।@I63.com

\begin{abstract}
To develop an alternative treatment for lung cancer, a combination of two potent chemotherapeutic agents - liposomal apigenin and tyroservatide - was developed. The therapeutic potential of this combination was investigated using A549 cells. Apigenin and tocopherol derivative-containing D-alpha-tocopheryl polyethylene glycol 1000 succinate (TPGS) liposomes might improve the delivery of apigenin to tumor cells, both in vitro and in vivo. Importantly, compared to either agent alone, the combination of apigenin TPGS liposomes and tyroservatide exhibited superior cytotoxicity, induced stronger G2 arrest, and suppressed A549 cancer cell invasion at a lower dose. The proapoptotic synergistic effects were also observed in A549 cells using terminal deoxynucleotidyl transferase-mediated dUTP nick-end labeling, flow cytometry, and Western blot analysis. More importantly, in vivo results showed that the combination of apigenin TPGS liposomes and tyroservatide exhibited tumor-growth inhibitory effects in A549 cell-bearing mice. In conclusion, our study showed that this combination therapy could serve as a promising synergistic therapeutic approach to improve outcomes in patients with lung cancer. Keywords: apigenin, TPGS liposomes, tyroservatide, synergistic antitumor, apoptosis
\end{abstract}

\section{Introduction}

Cancer is considered a public health problem caused by complex underlying mechanisms. Lung cancer is known as the leading cause of cancer-related deaths worldwide. ${ }^{1}$ Chemotherapeutic agents are considered central in the clinical management of lung cancer. ${ }^{2,3}$ Multidrug resistance and non-selective toxicity have been recognized as major problems associated with chemotherapeutic antitumor agents. ${ }^{46}$

Apigenin - a flavonoid extracted from vegetables and fruits, such as celery, parsley, and oranges - exhibits promising antitumor potential against lung cancer. It has many biochemical activities, including targeting of the phosphoinositide 3-kinase (PI3K)/ Akt signaling pathway, upregulation of tumor necrosis factor (TNF)-related apoptosisinducing ligand (TRAIL) death receptors DR4/DR5 expression in a p53-dependent manner, inhibition of glutamine metabolism, and induction of the epidermal growth factor receptor (EGFR) pathway. ${ }^{7-10}$ However, the clinical use of apigenin is restricted, owing to its poor biopharmaceutical properties, including water insolubility and poor absorption. ${ }^{11}$ Although apigenin is predicted to be safe for lung cancer treatment, similar to the majority of antitumor drugs, resistance could develop during the course of treatment, which could result in therapeutic failure. These drawbacks could be overcome by using a liposomal form of apigenin and combining it with other chemotherapeutic drugs. 
Liposomes - spherical vesicles composed of a phospholipid bilayer membrane and an aqueous core - are widely accepted as passive targeted drug-delivery systems with enhanced membrane permeability and improved retention. ${ }^{12,13}$ Several commercial liposome-based products are used clinically. ${ }^{14-18}$ D-alpha-tocopheryl polyethylene glycol 1000 succinate (TPGS) is formed from a water-soluble polyethylene glycol 1000-esterified derivative of vitamin E succinate, and has been used as a component of nanocarriers because it acts as a solubilizer, inhibits P-glycoprotein-mediated multidrug resistance, and enhances the bioavailability of anticancer drugs. ${ }^{19-23}$ Therefore, apigenin TPGS-coated liposomes were designed to increase the solubility, absorption, and retention, as well as to enhance the antitumor effects of apigenin.

Cancer treatment relying on a single antitumor drug does not usually result in satisfactory outcomes. Recent anticancer therapy using drug combinations has been found to be successful in clinical practice; thus, it is considered a promising strategy for enhancing the therapeutic efficacy. ${ }^{24,25}$ Moreover, synergistic anticancer drug combinations may counteract drug resistance, reduce the dose required, and enhance the antitumor effects. Therefore, cocktail therapy has been increasingly used as a primary cancer treatment regimen to avoid the poor response and resistance that is often encountered with monotherapy. ${ }^{8,26,27}$ Tyroservatide (YSV) is a bioactive tripeptide (tyrosyl-seryl-valine) obtained from the spleen of pigs. It has been found to affect integrin-focal adhesion kinase signal transduction and inhibit histone deacetylase activity. YSV has been shown to exhibit antitumor effects in lung cancer. ${ }^{28-31}$ Therefore, a combination of YSV and apigenin liposomes may reduce chemoresistance.

In the present study, we aimed to compare the effectiveness of apigenin-loaded TPGS-coated liposomes with that of free apigenin. In addition, we investigated the potential synergistic effects of the combination of apigenin liposomes and YSV, and compared the antitumor efficacy of this combination with that of free apigenin and YSV.

\section{Materials and methods}

\section{Materials, cell lines, and animals}

YSV was synthesized by Shanghai Ziyu Biotech Co, Ltd. (Shanghai, People's Republic of China). Injectiongrade yolk lecithin PC-98T was purchased from Shanghai Advanced Vehicle Technology Ltd. Co. (Shanghai, People's Republic of China). TPGS was obtained from Sigma-Aldrich (St Louis, MO, USA). Apigenin, coumarin 6 (C-6), 4',6diamidino-2-phenylindole (DAPI), and 1'-dioctadecyl-3,3,3', 3'-tetramethylindotricarbocyanine iodide (DiR) were purchased from Aladdin Scientific Inc. (Shanghai, People's Republic of China). Antibodies against caspase 3, caspase 8, caspase 9, poly (ADP-ribose) polymerase (PARP), Bax, and Bcl-2 were purchased from Nanjing KeyGen Biotech. Co. Ltd. (Nanjing, People's Republic of China). Fetal bovine serum (FBS) and Roswell Park Memorial Institute (RPMI) 1640 medium were obtained from Gibco-BRL (Gaithersburg, MD, USA). All other reagents were of chromatography grade. A549 cells were supplied by Nanjing KeyGen Biotech. Inc. (Nanjing, People's Republic of China) and grown in RPMI 1640 media containing $\mathrm{FBS}$ at $37^{\circ} \mathrm{C}$ in $5 \% \mathrm{CO}_{2}$ atmosphere.

Male nude mice were purchased from the SLEK Lab Animal Center of Shanghai (Shanghai, People's Republic of China), and received care according to the Guidelines on the Use of Laboratory Animals. All animal experiments were performed in accordance with the principles of care and use of laboratory animals, and were approved by the experimental animal administrative committee of Suqian Branch Jiangsu Province Hospital. Ethical and legal approval was obtained prior to the study. The nude mice were subcutaneously inoculated with A549 cells ( $5 \times 10^{6}$ cells/mouse); tumor size was measured by a Vernier caliper, and the tumor volume $(V)$ was calculated as follows: $V=a \times b^{2} / 2$, where $a$ represents the largest diameter, and $b$ is the smallest one.

\section{Preparation of apigenin-loaded TPGS liposomes}

Apigenin-loaded TPGS liposomes were prepared as previously described, but with a slight modification. ${ }^{32}$ Apigenin, yolk lecithin, and TPGS were dissolved in absolute alcohol, and the solution was evaporated under vacuum. The resultant thin film was hydrated with pure water for $12 \mathrm{~h}$. After complete lipid hydration and formation of liposomes, the coarse dispersion was sonicated using a probe sonicator to obtain the apigenin-loaded TPGS liposomes; C-6 liposomes and DiR liposomes were also prepared using the same method.

\section{Characterization of apigenin-loaded TPGS liposomes}

The average size and zeta potential were measured by dynamic light scattering (Mastersizer 2000, Malvern, UK) at a sample temperature of $25^{\circ} \mathrm{C}$ after equilibration for $5 \mathrm{~min}$. All liposome dispersions were diluted with distilled water (1:10) prior to the measurements.

The morphology of liposomes was investigated by transmission electron microscopy (TEM, JEM 100CX; JEOL, Tokyo, Japan). Apigenin-loaded TPGS liposomes were 
dropped on copper netting and stained with phosphomolybdic acid for morphological observation.

\section{Cellular uptake and intracellular localization analysis}

A549 cells were seeded and cultured at $37^{\circ} \mathrm{C}$ for $24 \mathrm{~h}$. C-6, which can be encapsulated into various nanocarriers, was used as a model fluorescent molecule to evaluate the cellular uptake efficiency of the liposomes.

After treatment with C-6 or C-6 TPGS liposomes for $4 \mathrm{~h}$, cells were washed with cold phosphate-buffered saline (PBS) thrice and immobilized with absolute ethyl alcohol for $10 \mathrm{~min}$. Then, the cell nuclei were counterstained with DAPI and observed under a fluorescence microscope (IX71; Olympus Corp., Tokyo, Japan) or confocal laser scanning microscope (CLSM; Olympus Corp.).

\section{In vivo bioluminescence imaging}

When the tumor volume of A549-bearing male nude mice reached $\sim 100 \mathrm{~mm}^{3}$, DiR or DiR TPGS liposomes were injected into the tail vein. The distribution of fluorescence was observed after injection to examine the targeting effect of TPGS liposomes. After imaging for $12 \mathrm{~h}$, the nude mice were sacrificed, the major organs and tumors were harvested, and fluorescence images were captured.

\section{MTT assay}

A549 cells were seeded in a 96-well microtiter plate and cultured overnight. Then, the cells were treated with serial concentrations of apigenin, YSV, and apigenin plus YSV for $24 \mathrm{~h}$. Cell viability was evaluated as described previously. ${ }^{33}$

\section{Cell-cycle analysis}

Cells were seeded into six-well plates and incubated. After treatment with apigenin, YSV, or a combination of both for $24 \mathrm{~h}$, cells were harvested and stained with propidium iodide in the dark for analysis by flow cytometry.

\section{Wound-healing assays}

The cell monolayer formed in a 24-well plate was scratched using a $200 \mu \mathrm{L}$ pipette tip, washed three times with PBS, and then incubated in culture media that contained apigenin, YSV, or a combination of both for $24 \mathrm{~h}$. The invasion potential was investigated using microscopy.

\section{Cell apoptosis assay}

DNA fragmentation was observed by terminal deoxynucleotidyl transferase-mediated dUTP nick-end labeling (TUNEL) assay $24 \mathrm{~h}$ after treatment. A549 cells were treated with proteinase $\mathrm{K}$ for $30 \mathrm{~min}$ at $37^{\circ} \mathrm{C}$ to remove the remaining nucleases. The cytoblasts were stained with the TUNEL reaction solution in a dark room. Apoptotic cytoblasts and normal nuclei were labeled brown and purple, respectively. Both were photographed for morphological examination.

For quantitative analysis of the apoptotic ratio after treatment with apigenin, YSV, and a combination of both for 24 h, A549 cells were trypsinized and resuspended in PBS. Then, the cells were analyzed by flow cytometry using an Annexin V-FITC apoptosis detection kit.

For analysis of apoptotic protein expression after treatment with apigenin, YSV, and a combination of both for $24 \mathrm{~h}$, total protein was isolated after cell treatment with a lysis buffer. Skimmed milk was used to block nonspecific membrane protein binding. Samples were incubated with the primary antibodies overnight at $4{ }^{\circ} \mathrm{C}$, and then incubated with the secondary antibodies. The proteins were quantified using Western blot analysis.

\section{Antitumor efficacy in vivo}

The antitumor efficacy of apigenin, YSV, and a combination of both was investigated in a xenograft model using nude mice. Mice were randomly divided into five groups $(\mathrm{n}=5)$ when the tumor volumes were $\sim 50 \mathrm{~mm}^{3}$. The mice were intravenously treated with apigenin $(2.7 \mu \mathrm{g} / \mathrm{kg}), \mathrm{YSV}$ $(270 \mu \mathrm{g} / \mathrm{kg})$, or a combination, via the tail vein, every 3 days. Tumor volumes and body weight were recorded every 3 days. Mice were sacrificed 15 days after the initial injection. Tumors were excised for histopathological analysis using hematoxylin and eosin (H\&E) staining.

\section{Data analysis}

Data are expressed as the means \pm standard deviation. Synergism was evaluated using the CompuSyn software with Chou-Talalay plotting. $P$-values $<0.05$ were considered statistically significant.

\section{Results and discussion Characterization of apigenin-loaded TPGS liposomes}

The average size, zeta potential, and morphology of apigeninloaded TPGS liposomes are shown in Figure 1. The size of apigenin-loaded TPGS liposomes was $118.6 \pm 8.1 \mathrm{~nm}$, with a uniform distribution. The apigenin-loaded TPGS liposomes had a negative zeta potential of $-6.8 \pm 0.28 \mathrm{mV}$ owing to the presence of surface TPGS. As shown in Figure 1C, apigenin-loaded TPGS liposomes existed as homogeneous 
A

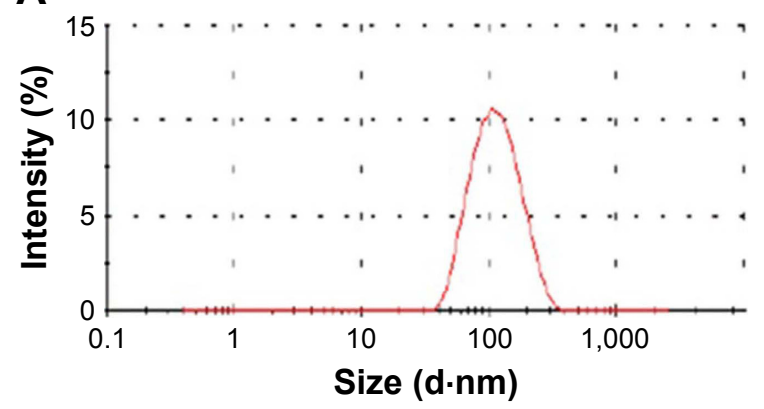

B

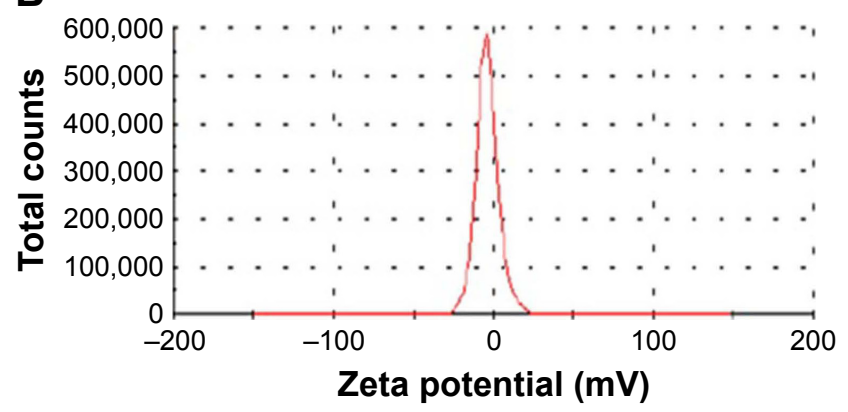

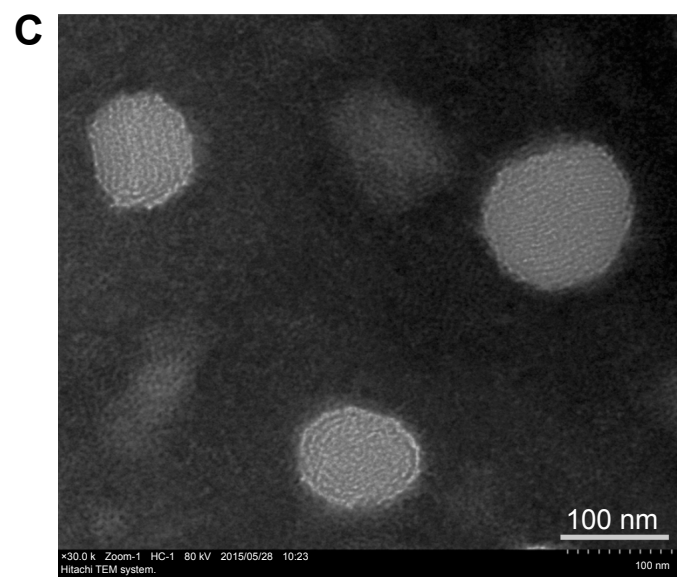

Figure I The average size (A), zeta potential (B), and morphology $(\mathbf{C})$ of apigenin-loaded TPGS liposomes $(n=3)$. Abbreviation: TPGS, D-alpha-tocopheryl polyethylene glycol 1000 succinate.

spheres with a mean diameter of $\sim 100 \mathrm{~nm}$. The liposome size observed by TEM was consistent with that measured by dynamic light scattering.

\section{Cellular uptake and intracellular distribution of TPGS liposomes}

After incubation for $4 \mathrm{~h}$ at $37^{\circ} \mathrm{C}$, green fluorescence was observed in C-6 and C-6 TPGS liposome-treated cells, with the latter showing more intense fluorescence (Figure 2A), which indicated an increased amount of C-6 uptake with C-6 TPGS liposomes. To further elucidate the permeability-enhancing effects of TPGS liposomes, intracellular localization was examined, as shown in Figure 2B. Most of the C-6 was internalized and distributed through the cytoplasm, showing that TPGS liposomes were successfully taken up by A549 cells.

\section{In vivo fluorescence imaging studies}

Free DiR and DiR-labeled TPGS liposomes were injected into the tail vein of nude mice to evaluate the targeting effect of TPGS liposomes. As shown in Figure 2C, the DiR TPGS liposomes started to accumulate in tumor tissues $4 \mathrm{~h}$ after injection. The fluorescent signal in the tumor sites reached the maximum intensity at $8 \mathrm{~h}$. Subsequently, the fluorescence intensity gradually decreased; however, it remained until $12 \mathrm{~h}$. The ex vivo data showed that the tumor, liver, and spleen retained fluorescence for $12 \mathrm{~h}$. It could be inferred that TPGS-coated liposomes delivered the antitumor drug directly into the tumor, and was retained for a longer period than that delivered through passive diffusion. ${ }^{34}$ Thus, TPGScoated liposomal delivery of anticancer drugs would enhance their antitumor efficacy.

\section{Evaluation of the combination effect of apigenin and YSV in A549 cells}

Before investigation of the combined effects of apigenin and YSV therapy, we evaluated the cytotoxicity of apigenin and YSV monotherapy in the A549 cell line using an MTT assay. Then, the cytotoxicity of different molar combinations of apigenin and YSV was evaluated. Our results showed that, at a concentration of $2.7 \mu \mathrm{g} / \mathrm{mL}$, apigenin exhibited weak antitumor effects in A549 cells. When A549 cells were treated with apigenin plus YSV (apigenin, $2.7 \mu \mathrm{g} / \mathrm{mL}$; YSV, $270 \mu \mathrm{g} / \mathrm{mL}$ ), $>50 \%$ tumor inhibition was observed. However, in the monotherapy treatment groups, $<20 \%$ inhibition was observed at the same concentrations (Figure $3 \mathrm{~A}$ and B). Thus, it could be inferred that the combination of apigenin 
A
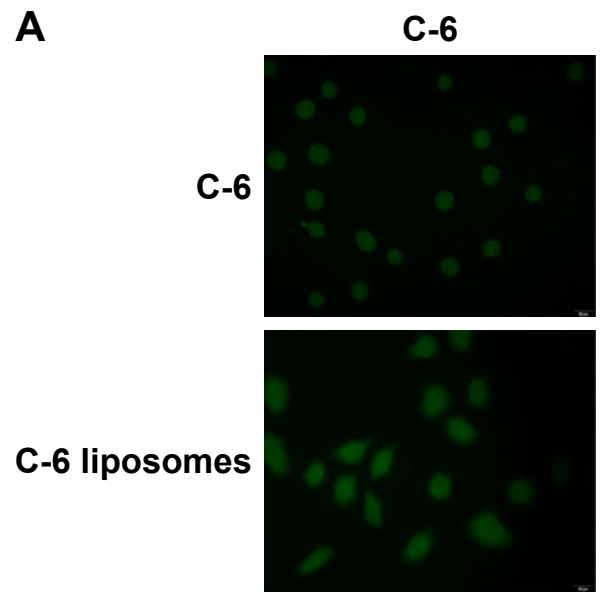

B
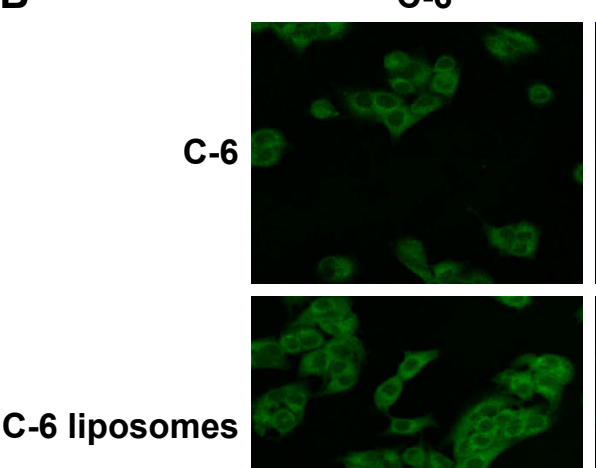
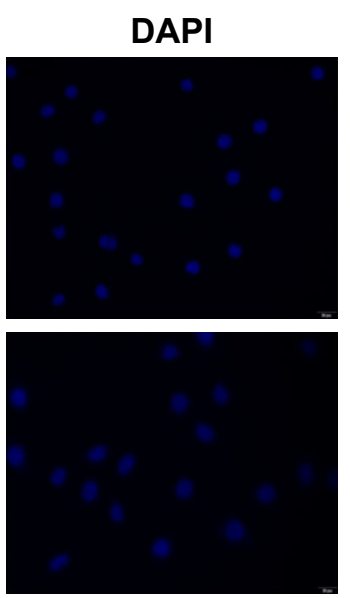

DAPI
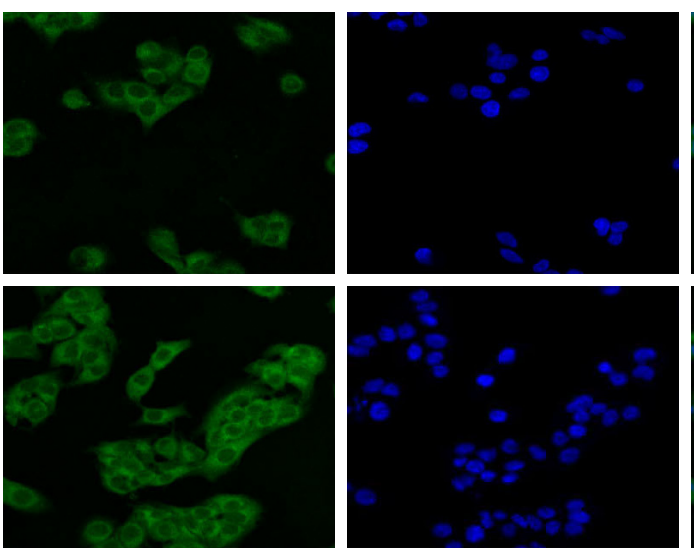

$8 \mathrm{~h}$

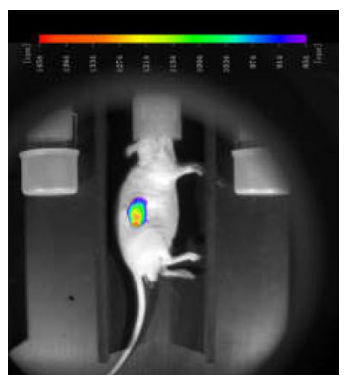

Merged

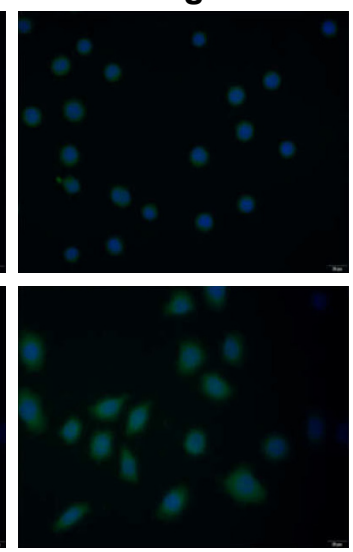

Merged
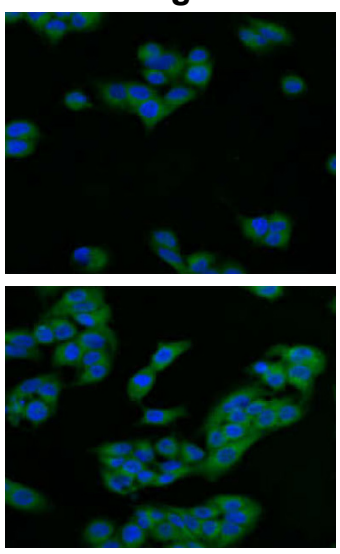

C

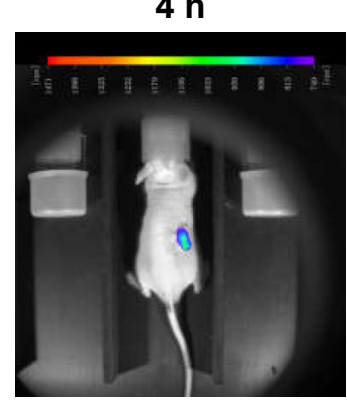

$12 \mathrm{~h}$

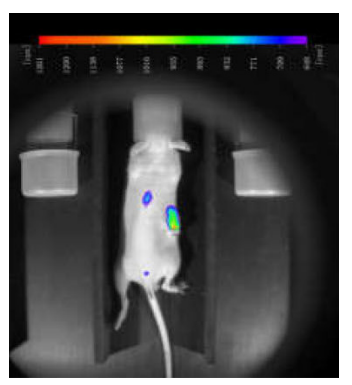

Tissues

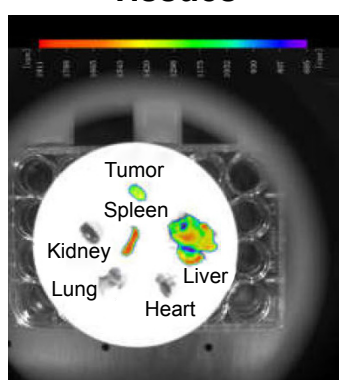

Figure 2 Cellular uptake (A), intracellular distribution (B), and in vivo imaging $(\mathbf{C})$ of TPGS liposomes $(n=3)$. Abbreviation: TPGS, D-alpha-tocopheryl polyethylene glycol 1000 succinate.

and YSV exhibited synergistic antitumor effects. To assess whether apigenin and YSV combination at a ratio of 1:100 exhibited synergistic effects against lung cancer, we performed a Chou-Talalay analysis. ${ }^{35,36}$ Our results showed that the combination index (CI) of apigenin and YSV was less than 1 at an apigenin:YSV ratio of $1: 100$. The $\mathrm{CI}$ for apigenin $(0.34,0.68,1.35,2.7$, and $5.4 \mu \mathrm{g} / \mathrm{mL})$ combination treatment was found to be $<1$ ( $\mathrm{CI}=0.68,0.65,0.54,0.53$, and 0.52 , respectively), suggesting that this combination interacted synergistically at a ratio of 1:100 (Figure 3C). Therefore, apigenin at $2.7 \mu \mathrm{g} / \mathrm{mL}$ and $\mathrm{YSV}$ at $270 \mu \mathrm{g} / \mathrm{mL}$ (ratio $=1: 100$ ) were selected as optimal doses for combination therapy in the subsequent experiments.

\section{Synergistic effects on cell cycle}

The cell-cycle distribution was assayed using flow cytometric analysis. There was an increase in the $\mathrm{G}_{2} / \mathrm{M}$ phase fraction in the A549 cells treated with either apigenin or YSV alone, compared to that in the control cells (Figure 4A). Moreover, combination therapy significantly increased the fraction of cells in $\mathrm{G}_{2} / \mathrm{M}$ phase, compared to those treated with either apigenin or YSV alone. When apigenin TPGS liposomes 

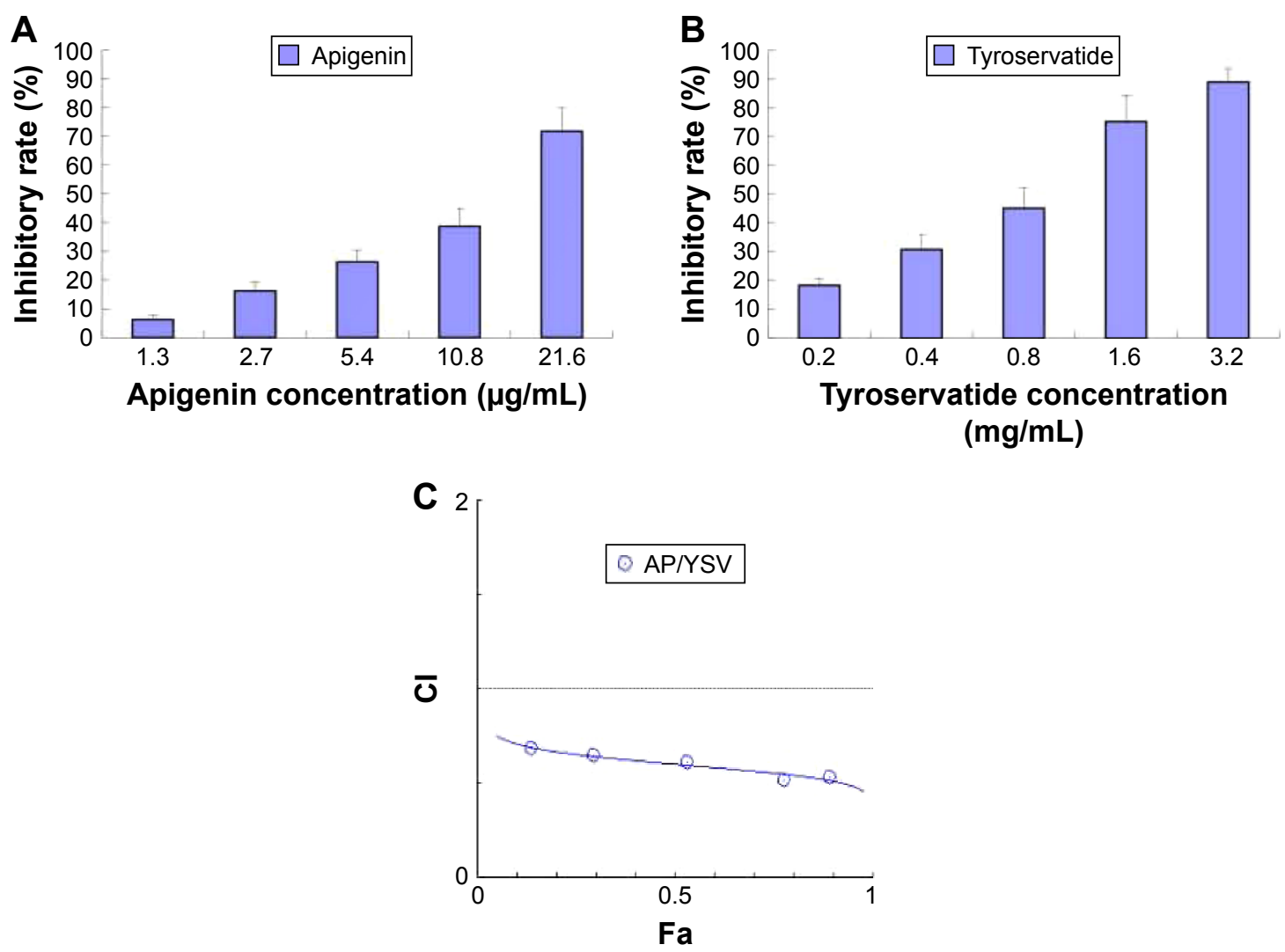

Figure 3 MTT assay of apigenin (A), YSV (B) monotherapy, and a Chou-Talalay plot of the combination effect (C) in A549 cells ( $n=5)$. Abbreviations: $\mathrm{Cl}$, cooperativity index; Fa, fraction affected.

A
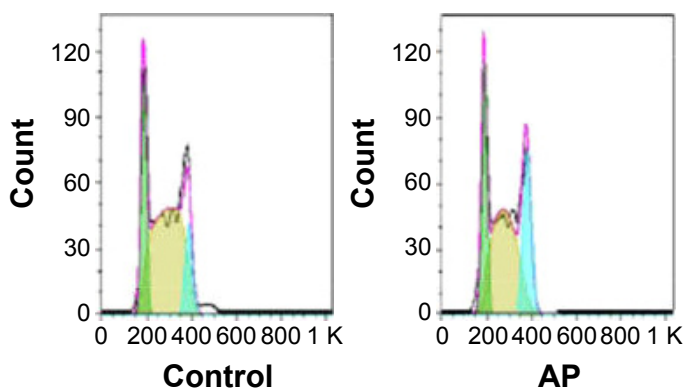

Control

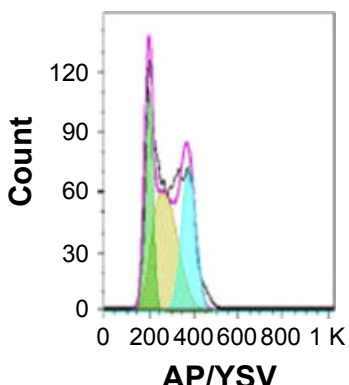

AP

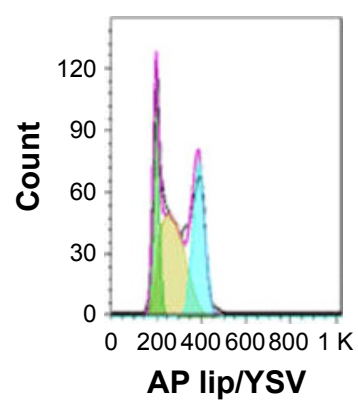

B

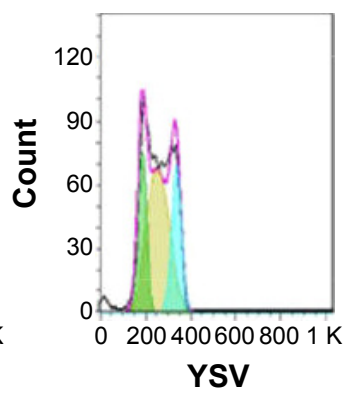

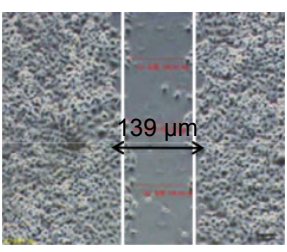

AP

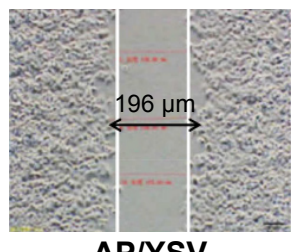

AP/YSV

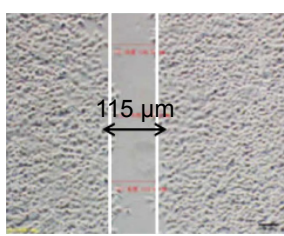

Control

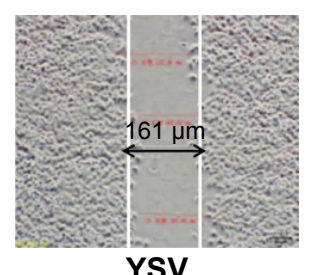

YSV

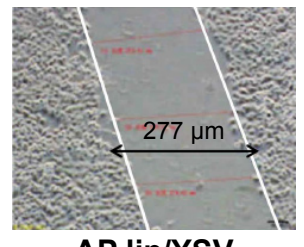

Figure 4 Synergistic effects of apigenin-loaded TPGS liposomes and YSV on cell cycle (A) and invasion (B) in A549 cells ( $n=3$ ) Abbreviation: TPGS, D-alpha-tocopheryl polyethylene glycol 1000 succinate. 
were used, the $\mathrm{G} 2 / \mathrm{M}$ population increased dramatically from $28.4 \%$ to $38.6 \%$, compared to that of the combination of free apigenin and YSV, which could be attributable to the intracellular accumulation of apigenin delivered from the TPGS liposomes $(P<0.05)$.

\section{Effect on cell invasion}

A wound-healing assay was used to evaluate the invasive potential in the presence of the test agents. The results revealed that both apigenin and YSV individually exerted an inhibitory effect on cancer cell invasion. However, the anti-invasive effects of apigenin and YSV combination were greater than that of either apigenin or YSV alone $(P<0.05)$. The cell-cell distance was maximal after treatment with apigenin TPGS liposomes plus YSV, compared to the other groups (Figure 4B).

\section{Proapoptotic effects of the combination treatment in A549 cells}

Next, we investigated whether apigenin TPGS liposomes and YSV combination exerted synergistic proapoptotic effects. Qualitative and quantitative apoptosis analyses were performed using inverted microscopy, flow cytometry, and Western blot analysis. When cells were exposed to a combination of apigenin TPGS liposomes and YSV, apoptotic morphology - mainly, chromatin edge fog, membrane aggregation, and nuclear dimpling - was observed (Figure 5A). In the control and apigenin only-treated groups, TUNELpositive apoptotic cells were distributed sporadically, whereas in the other groups, the number of TUNEL-positive apoptotic cells greatly increased $(P<0.05)$. Interestingly, the number of apoptotic cells was larger in the apigenin TPGS liposomes and YSV combination-treated group, compared to the apigenin and YSV combination group $(P<0.05)$, suggesting that the liposome-enhanced uptake of apigenin increased apoptosis of A549 cells.

In addition, flow cytometry was performed to distinguish between apoptosis and necrosis. As shown in Figure 5B, apigenin, YSV, and the apigenin and YSV combination induced $5.1 \%, 10.3 \%$, and $19.6 \%$ apoptosis, which indicated that the combination therapy resulted in a 3.84- and 1.91-fold increase in apoptosis, compared to apigenin and YSV alone $(P<0.05)$, respectively. These data showed that apigenin and YSV exhibited synergic proapoptotic effects. Moreover, the combination of apigenin TPGS liposomes and YSV induced 28.9\% A549 cell apoptosis, with a 1.47 -fold increase compared to the combination of free apigenin and YSV $(P<0.05)$. Therefore, the enhanced uptake of apigenin delivered by TPGS liposomes combined with YSV increased cell apoptosis, which might further result in greater antitumor efficacy.

Expression levels of the key regulator proapoptotic proteins were also measured by Western blot analysis. Combined apigenin and YSV elicited higher expression levels of the key regulator proapoptotic proteins, compared to the individual agents, demonstrating the stronger effects of the combination therapy. In addition, results showed that Bcl-2 levels decreased, whereas Bax, caspase 3, caspase 8, caspase 9, and PARP levels significantly increased. Moreover, Bax/ Bcl-2 ratio significantly increased after apigenin TPGS liposomes and YSV combination treatment (Figure 5C). Taken together, the apigenin TPGS liposomes and YSV combination exhibited higher efficacy, compared to free apigenin and the YSV combination.

\section{In vivo antitumor activity}

To confirm whether this combination could have an impact on the efficacy of lung cancer treatment, the efficiency of the synergistic antitumor combinations was tested in vivo using A549 tumor-bearing nude mice. As shown in Figure 6A and B, regarding the individual agents, apigenin had a slightly better antitumor activity at low concentrations; in addition, tumor volumes in the YSV group were significantly smaller than those in the saline-treated group $(P<0.05)$. The combination of apigenin and YSV showed stronger antitumor effects than either monotherapy, confirming the synergistic effects of the combination. Apigenin TPGS liposomes and YSV combination exhibited the strongest antitumor effects $(P<0.05)$, which suggested that TPGS liposomal delivery system was superior. There was no change in body weight of the mice, which indicated that the formulations were safe.

To further confirm the synergistic antitumor effects, H\&E staining of tumor slices was performed to assess tumor cell death. ${ }^{37}$ Both the saline- and apigenin-treated groups showed a large number of living cancer cells that appeared blue. The combination of apigenin and YSV induced significant cancer cell necrosis, which appeared pink, compared to treatment with each individual agent. The combination treatment of apigenin TPGS liposomes and YSV showed the strongest necrotic effects, indicating improved synergism provided by the TPGS liposomal delivery system (Figure 6C).

\section{Conclusion}

The present study aimed to study the potential applications of apigenin TPGS liposomes and YSV combination as 

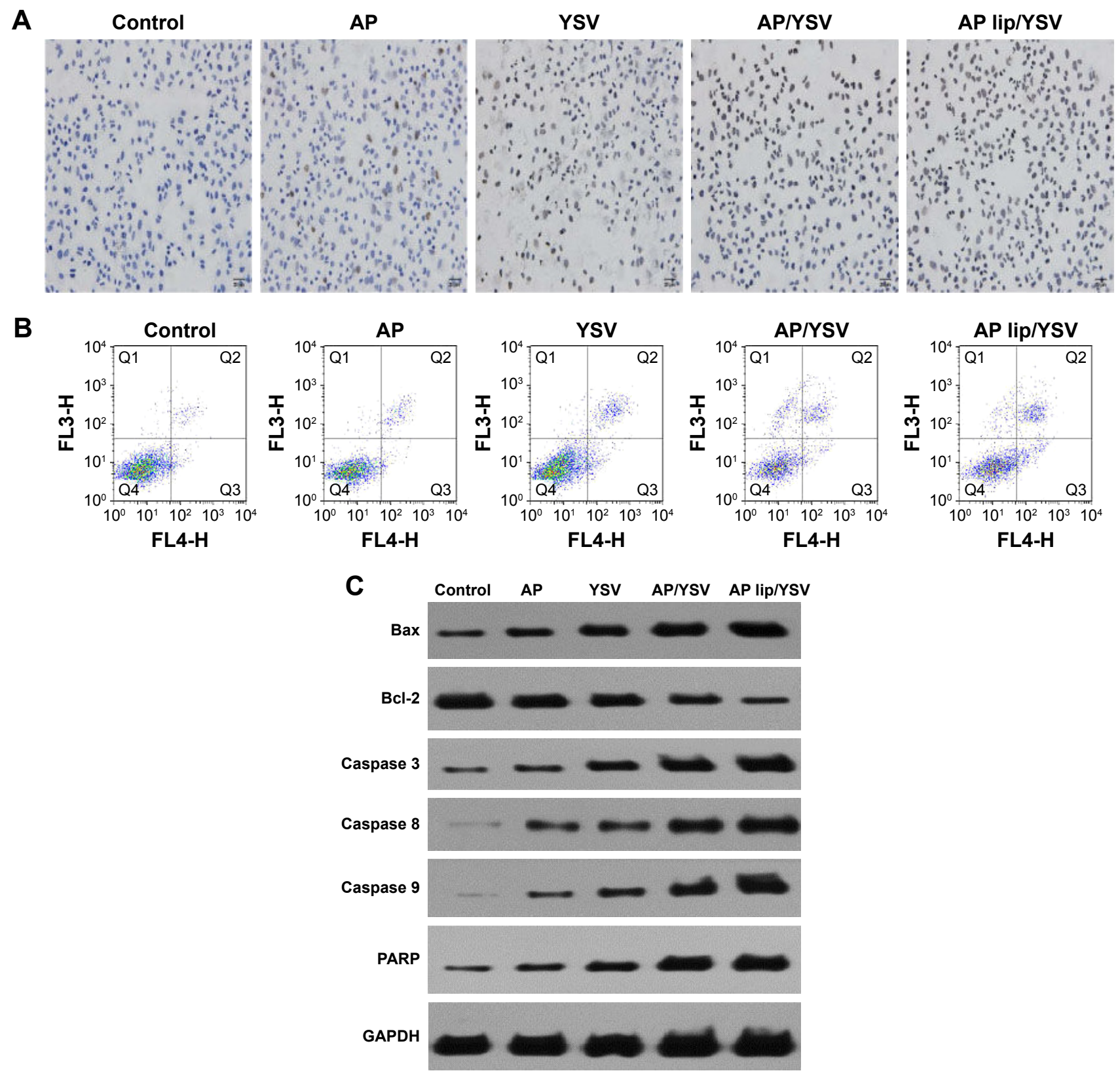

Figure 5 TUNEL (A), Annexin V-FITC apoptosis detection (B), and Western blot analysis (C) of apigenin-loaded TPGS liposomes and YSV in vitro in A549 cells ( $\mathrm{n}=3$ ). Abbreviations: FITC, fluorescein isothiocyanate; TPGS, D-alpha-tocopheryl polyethylene glycol 1000 succinate.

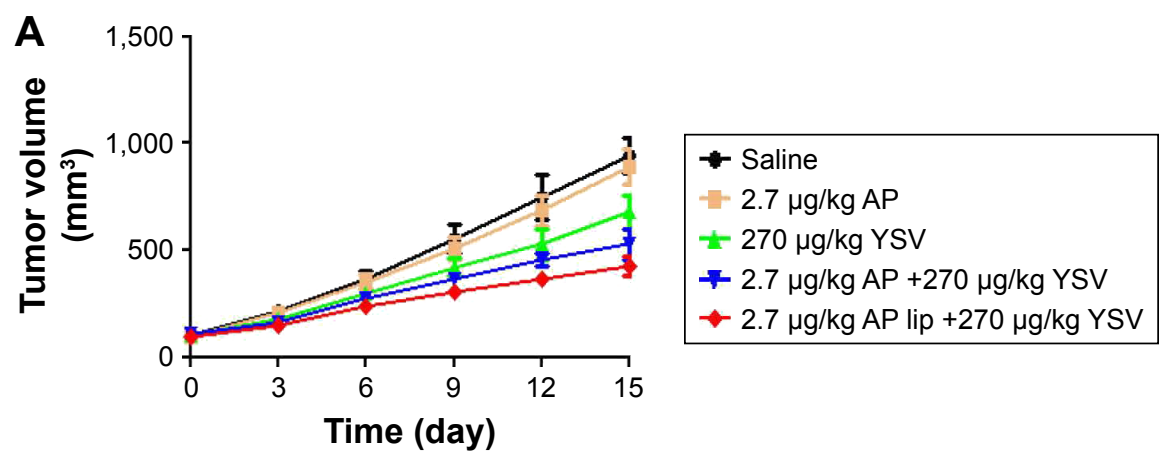

Figure 6 (Continued) 

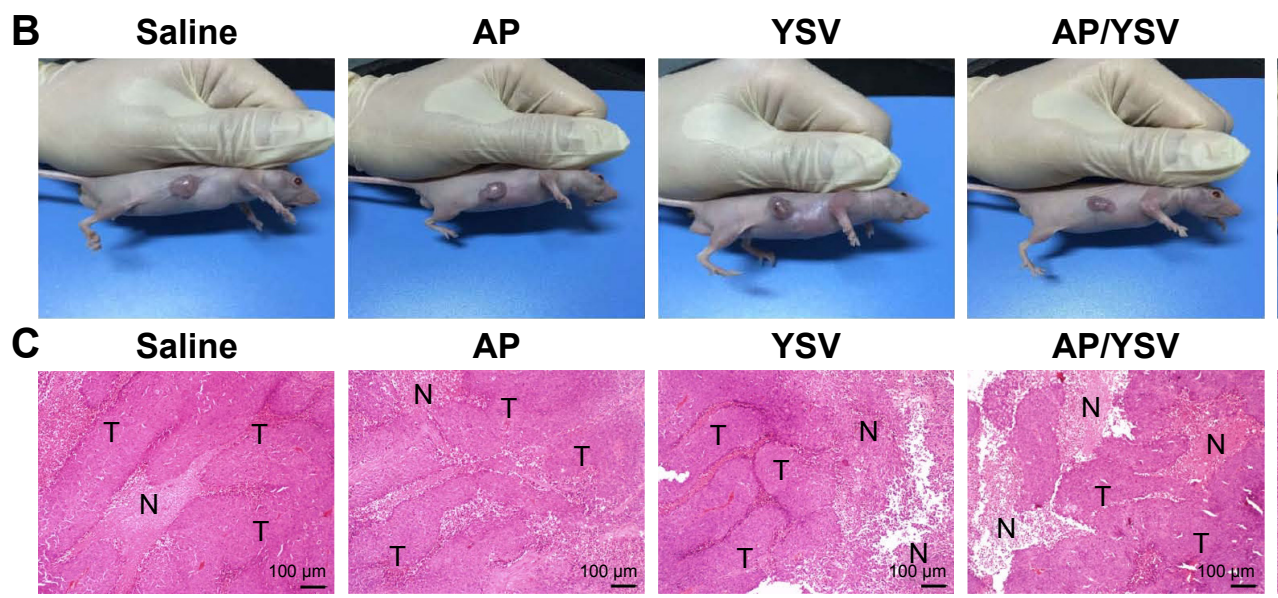

AP lip/YSV
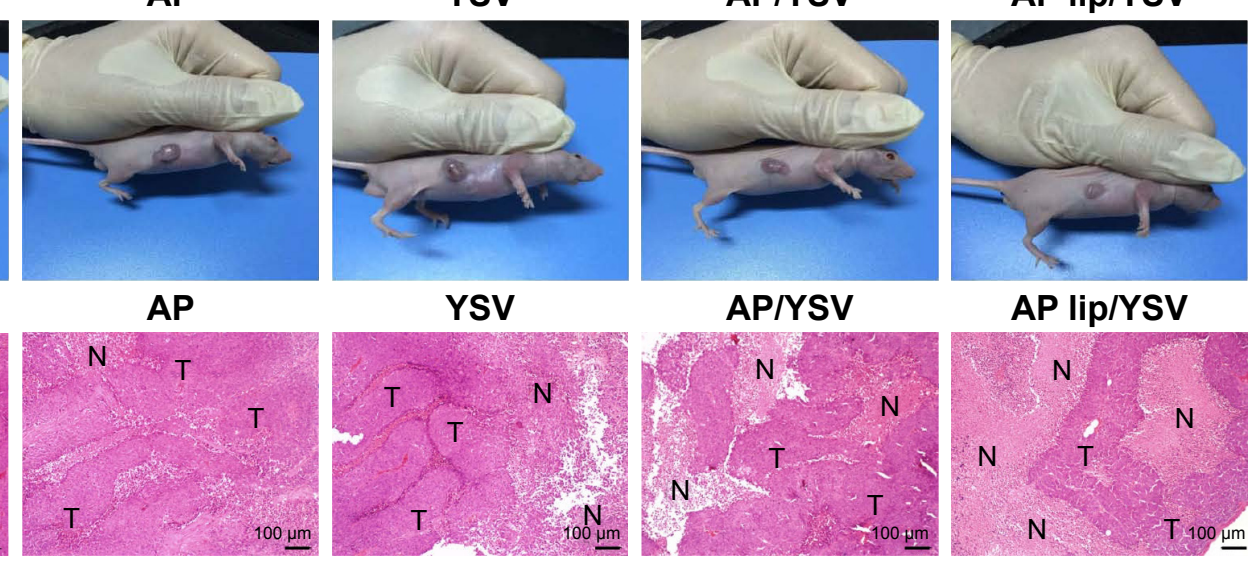

Figure 6 Curves of tumor volumes (A), morphology (B), and typical H\&E-stained images (C) ('N' represents necrosis area, 'T' represents tumor mass) on day I5 after injection of the indicated formulations into the tail vein $(n=5)$.

anticancer therapy. We showed that cocktail therapy using a combination of apigenin TPGS liposomes and YSV, which exhibited significant and synergistic proapoptotic effects, could provide an effective chemotherapeutic regimen for lung cancer treatment. MTT assay showed that low concentrations of apigenin exerted low in vitro cytotoxicity in A549 cancer cells. Imaging of cellular uptake in vitro and in vivo suggested that TPGS liposomes enhanced tumor targeting and apigenin uptake by tumor cells. Cocktail therapy using apigenin TPGS liposomes and YSV exhibited significantly stronger proapoptotic effects in vitro and in vivo, compared to that of apigenin and YSV either alone or in combination. Therefore, the combination of apigenin TPGS liposomes and YSV could be useful in lung cancer treatment in the future.

\section{Acknowledgment}

This work was supported by the National Natural Science Foundation of China (Grant no 81403119), 333 project of Jiangsu Province (BRA2016258), and the Science and Technology Support Project of Suqian.

\section{Disclosure}

The authors report no conflicts of interest in this work.

\section{References}

1. Siegel RL, Miller KD, Jemal A. Cancer statistics, 2017. CA Cancer J Clin. 2017;67(1):7-30.

2. Tangutur AD, Kumar D, Krishna KV, Kantevari S. Microtubule targeting agents as cancer chemotherapeutics: an overview of molecular hybrids as stabilising and destabilising agents. Curr Top Med Chem. Epub 2017 Jan 4.

3. Wijdeven RH, Pang B, Assaraf YG, Neefjes J. Old drugs, novel ways out: drug resistance toward cytotoxic chemotherapeutics. Drug Resist Updat. 2016;28:65-81.

4. Walls GM, Lyon AR, Harbinson MT, Hanna GG. Cardiotoxicity following cancer treatment. Ulster Med J. 2017;86(1):3-9.
5. Papadas A, Asimakopoulos F. Mechanisms of resistance in multiple myeloma. Handb Exp Pharmacol. Epub 2017 March 18.

6. Colmegna B, Morosi L, D'Incalci M. Molecular and pharmacological mechanisms of drug resistance: an evolving paradigm. Handb Exp Pharmacol. Epub 2017 Mar 23.

7. Zhou Z, Tang M, Liu Y, Zhang Z, Lu R, Lu J. Apigenin inhibits cell proliferation, migration, and invasion by targeting Akt in the A549 human lung cancer cell line. Anticancer Drugs. 2017;28(4):446-456.

8. Chen M, Wang X, Zha D, et al. Apigenin potentiates TRAIL therapy of non-small cell lung cancer via upregulating DR4/DR5 expression in a p53-dependent manner. Sci Rep. 2016;6:35468.

9. Lee YM, Lee G, Oh TI, et al. Inhibition of glutamine utilization sensitizes lung cancer cells to apigenin-induced apoptosis resulting from metabolic and oxidative stress. Int J Oncol. 2016;48(1):399-408.

10. Cheng CY, Hu CC, Yang HJ, Lee MC, Kao ES. Inhibitory effects of scutellarein on proliferation of human lung cancer A549 cells through ERK and NFKB mediated by the EGFR pathway. Chin J Physiol. 2014; 57(4):182-187.

11. Zhang J, Liu D, Huang Y, Gao Y, Qian S. Biopharmaceutics classification and intestinal absorption study of apigenin. Int $J$ Pharm. 2012;436(1-2):311-317.

12. Castoldi A, Herr C, Niederstraßer J, et al. Calcifediol-loaded liposomes for local treatment of pulmonary bacterial infections. Eur $J$ Pharm Biopharm. Epub 2016 Nov 22.

13. Jøraholmen MW, Basnet P, Acharya G, Škalko-Basnet N. PEGylated liposomes for topical vaginal therapy improve delivery of interferon alpha. Eur J Pharm Biopharm. 2017;113:132-139.

14. Fouladi F, Steffen KJ, Mallik S. Enzyme-responsive liposomes for the delivery of anticancer drugs. Bioconjug Chem. 2017;28(4):857-868.

15. Lee Y, Thompson DH. Stimuli-responsive liposomes for drug delivery. Wiley Interdiscip Rev Nanomed Nanobiotechnol. Epub 2017 Feb 15.

16. Panahi Y, Farshbaf M, Mohammadhosseini M, et al. Recent advances on liposomal nanoparticles: synthesis, characterization and biomedical applications. Artif Cells Nanomed Biotechnol. 2017;45(4):788-789.

17. Chang HI, Yeh MK. Clinical development of liposome-based drugs: formulation, characterization, and therapeutic efficacy. Int J Nanomedicine. 2012;7:49-60.

18. Bozzuto G, Molinari A. Liposomes as nanomedical devices. Int $J$ Nanomedicine. 2015;10:975-999.

19. Duhem N, Danhier F, Préat V. Vitamin E-based nanomedicines for anti-cancer drug delivery. J Control Release. 2014;182:33-44.

20. Guo Y, Luo J, Tan S, Otieno BO, Zhang Z. The applications of Vitamin E TPGS in drug delivery. Eur J Pharm Sci. 2013;49(2):175-186.

21. Zhang Z, Tan S, Feng SS. Vitamin E TPGS as a molecular biomaterial for drug delivery. Biomaterials. 2012;33(19):4889-4906. 
22. Shen R, Kim JJ, Yao M, Elbayoumi TA. Development and evaluation of vitamin E d- $\alpha$-tocopheryl polyethylene glycol 1000 succinate-mixed polymeric phospholipid micelles of berberine as an anticancer nanopharmaceutical. Int J Nanomedicine. 2016;11:1687-1700.

23. Hao J, Tong T, Jin K, et al. Folic acid-functionalized drug delivery platform of resveratrol based on Pluronic $127 / \mathrm{D}-\alpha$-tocopheryl polyethylene glycol 1000 succinate mixed micelles. Int J Nanomedicine. 2017; 12:2279-2292.

24. Zhang XY, Zhang PY. Combinations in multimodality treatments and clinical outcomes during cancer. Oncol Lett. 2016;12(6):4301-4304.

25. Bhaw-Luximon A, Jhurry D. Artemisinin and its derivatives in cancer therapy: status of progress, mechanism of action, and future perspectives. Cancer Chemother Pharmacol. 2017;79(3):451-466.

26. Cosenza M, Civallero M, Pozzi S, Marcheselli L, Bari A, Sacchi S. The combination of bortezomib with enzastaurin or lenalidomide enhances cytotoxicity in follicular and mantle cell lymphoma cell lines. Hematol Oncol. 2015;33(4):166-175.

27. Aras B, Yerlikaya A. Bortezomib and etoposide combinations exert synergistic effects on the human prostate cancer cell line PC-3. Oncol Lett. 2016;11(5):3179-3184.

28. Huang YT, Zhao L, Fu Z, et al. Therapeutic effects of tyroservatide on metastasis of lung cancer and its mechanism affecting integrin-focal adhesion kinase signal transduction. Drug Des Devel Ther. 2016; 10:649-663.

29. Xu Q, Lu R, Zhu ZF, et al. Effects of tyroservatide on histone acetylation in lung carcinoma cells. Int J Cancer. 2011;128(2):460-472.
30. Fu Z, Lu R, Jia J, et al. Inhibition of five xenografted human cancers and two murine cancers by the tripeptide tyroservatide. Anticancer Drugs. 2007;18(4):467-470.

31. Zheng M, Lu R, Che X, et al. Tyroservatide therapy for tumor growth, invasion and metastasis of Lewis lung carcinoma and human lung carcinoma A549. Oncology. 2006;70(6):418-426.

32. Jin X, Li M, Yin L, Zhou J, Zhang Z, Lv H. Tyroservatide-TPGSpaclitaxel liposomes: tyroservatide as a targeting ligand for improving breast cancer treatment. Nanomedicine. 2017;13(3):1105-1115.

33. Jin X, Zhang Y, Zhang Z, Che D, Lv H. Juglone loaded poloxamer 188/ phospholipid mixed micelles evaluated in vitro and in vivo in breast cancer. Int J Pharm. 2016;515(1-2):359-366.

34. Taurin S, Nehoff $\mathrm{H}$, Greish K. Anticancer nanomedicine and tumor vascular permeability; Where is the missing link? J Control Release. 2012;164(3):265-275.

35. Chou TC. Drug combination studies and their synergy quantification using the Chou-Talalay method. Cancer Res. 2010;70(2): 440-446.

36. Chou TC. Theoretical basis, experimental design, and computerized simulation of synergism and antagonism in drug combination studies. Pharmacol Rev. 2006;58(3):621-681.

37. Chen M, Zhang Y, Chen Z, Xie S, Luo X, Li X. Synergistic antitumor efficacy of redox and $\mathrm{pH}$ dually responsive micelleplexes for co-delivery of camptothecin and genes. Acta Biomater. 2017;49:444-455.
International Journal of Nanomedicine

\section{Publish your work in this journal}

The International Journal of Nanomedicine is an international, peerreviewed journal focusing on the application of nanotechnology in diagnostics, therapeutics, and drug delivery systems throughout the biomedical field. This journal is indexed on PubMed Central, MedLine, CAS, SciSearch $®$, Current Contents ${ }^{\circledR} /$ Clinical Medicine,

\section{Dovepress}

Journal Citation Reports/Science Edition, EMBase, Scopus and the Elsevier Bibliographic databases. The manuscript management system is completely online and includes a very quick and fair peer-review system, which is all easy to use. Visit http://www.dovepress.com/ testimonials.php to read real quotes from published authors. 